\title{
Thermodynamics of intercalated layer crystals at low temperatures
}

\author{
N.K.Tovstyuk* \\ Lviv National Ivan Franko University, Department of Physics, \\ 50 Dragomanov Str., 79005 Lviv, Ukraine
}

Received April 23, 2001

On the base of Anderson type model the density of electron states in the intercalated layer crystal is obtained. Thermodynamic stability depending on: band width, lamination degree, intercalant energy state and renormalized electron mixing, caused by intercalation, is analyzed.

Key words: thermodynamics, intercalated layer crystals, intercalant, intercalate

PACS: $65.90 .+i, 71.20 . T$

\section{Introduction}

All the layer crystals (LC) have common peculiarities, namely, anisotropy of chemical binding. It is very closely connected with processes of intercalation-deintercalation [1-3]. Due to these processes LC can be widely used in the systems of hydrogen accumulation, sources of high energy [2], superlattice structures [3]. To know the free energy changes is very important in understanding the thermodynamics of the intercalation processes [4-6]. Thus we study the dependence of free energy of an electron subsystem on the microscopic parameters describing the following processes:

(i) interlayer mixing in LC,

(ii) energy state of intercalant and its mixing with LC,

(iii) changes in electron mixing between the atoms of adjacent lattice layers close to the intercalate.

We assume the host material as the recipient of the guest species, the intercalate as the guest species resident in the host material, and the intercalant as the guest species impurities prior to intercalation [6].

*tose@polynet.lviv.ua 
The character of free energy changes (its sign) $\Delta F$ can be used as the test of thermodynamic advantage (or disadvantage) of the system at certain parameters, and thus, one can confirm the possibility or the impossibility of intercalation processes. Even in the case of thermodynamic disadvantage, the character of $\Delta F$ changes depending on i-iii parameters is important. Interlayer mixing stands out especially, because it can be easily changed under hydrostatic pressure or pressure along the anisotropic axis. Another opportunity is the choice of an atom-intercalant (ii). Theoretically, the system transformation from thermodynamic disadvantageous state to the advantageous one is possible in the case when there exists the tendency of $\Delta F$ decrease.

The value of $\Delta F$ is important, if the system "intercalated LC" is stable, because it determines the degree of intercalant-intercalate binding. The application of intercalation phenomenon is tightly connected with the deintercalation process, and thus $\Delta F$ magnitude determines energetic losses during the intercalation-deintercalation process.

\section{Theory}

We consider the changes in the density of electron states for the model layer crystal with layers in the XOY plane $[7,8]$. The atoms will be characterized by their radius-vectors with coordinates $(\vec{n}, \alpha)$ where $\vec{n}=\left(n_{x}, n_{y}\right)$; integer $\alpha$ fixes the layer number. We consider one-impurity one intercalate atom case. Let the impurity be located between land $l+1$ layers which is characterized by radius-vector $\vec{n}_{0}$.

In one-electron approximation in site representation, the Hamiltonian is as follows:

$$
\begin{aligned}
\hat{H}= & \sum_{\vec{n} \vec{n}^{\prime} \alpha \alpha^{\prime}} t\left\langle\vec{n} \alpha \mid \vec{n}^{\prime} \alpha^{\prime}\right\rangle c_{\vec{n} \alpha}^{+} c_{\vec{n}^{\prime} \alpha^{\prime}}+\left[\varepsilon_{0} a_{0}^{+} a_{0}+V_{0}\left(a_{0}^{+} c_{\vec{n}_{0} l}+\text { h.c. }+a_{0}^{+} c_{\vec{n}_{0} l+1}+\text { h.c. }\right)\right] \\
& -\left\{\sum_{\vec{n}}\left[t\langle\vec{n} l \mid \vec{n} l+1\rangle c_{\vec{n} l}^{+} c_{\vec{n} l+1}+\text { h.c. }\right]-\sum_{\vec{n}}\left[\gamma\langle\vec{n} l \mid \vec{n} l+1\rangle c_{\vec{n} l}^{+} c_{\vec{n} l+1}+\text { h.c. }\right]\right\},
\end{aligned}
$$

where the first sum describes the crystal lattice $\left(c_{n} \alpha\right.$ is Fermi operator of annihilation on $\vec{n}_{\alpha}$ site; the term in straight brackets is a sum of the energy of the electron on the intercalate and the energy of the electron overlapping of the intercalate with the nearest crystal atoms in the $l$ and $l+1$ layers; the term in figure brackets considers the change in overlapping from $t(n l / n l+1)$ to $\gamma(n l / n l+1)$, which is the result of the interlayer distance change at intercalation. As it was mentioned above, $t-\gamma$ can be both positive (in the case of intercalation dichalcogenide transition metals: organic molecules or $\mathrm{Li}$ in $\mathrm{TlGaS}_{2}$ ) or negative (in the case of intercalation alkali metals). 
Hamiltonian in momentum representation is of the following form $[7,8]$

$$
\begin{aligned}
\hat{H}= & \varepsilon_{0} a_{0}^{+} a_{0}+\sum_{\vec{\chi}, k} \varepsilon_{\vec{\chi} k} c_{\vec{\chi}, k}^{+} c_{\vec{\chi} k} \\
& +V_{0} \sum_{\vec{\chi}, k}\left[a_{0}^{+} c_{\vec{\chi} k} \mathrm{e}^{\mathrm{i} \vec{\chi} \vec{n}_{0}} \mathrm{e}^{\mathrm{i} k l}\left(1+\mathrm{e}^{\mathrm{i} k}\right)+c_{\vec{\chi} k}^{+} a_{0} \mathrm{e}^{-\mathrm{i} \vec{\chi} \vec{n}_{0}} \mathrm{e}^{-\mathrm{i} k l}\left(1+\mathrm{e}^{-\mathrm{i} k}\right)\right] \\
& -(t-\gamma) \sum_{\vec{\chi}_{1}, \vec{\chi}_{2}}\left[c_{\vec{\chi}_{1} k_{1}}^{+} c_{\vec{\chi}_{2} k_{2}} \mathrm{e}^{-\mathrm{i} k_{1} l} \mathrm{e}^{\mathrm{i} k_{2}(l+1)}+\text { h.c. }\right]
\end{aligned}
$$

here $\vec{\chi}=\left(k_{x}, k_{y}\right), k=k_{z}$. Electron dispersion law in the conduction band is equal to

$$
\varepsilon(\vec{\chi}, k)=\alpha \chi^{2}+t(1-\cos k) .
$$

Such a dispersion law reflects the difference between chemical bonds in different crystallographic directions of layer crystals. Normally to layers one can use tightbinding approximation because here the electrons are bound by weak overlapping of their atomic orbitals but in the layer the effective mass approximation is acceptable. Simultaneous choice of an effective mass approximation (for the electron motion in the layer plane) and the tight binding one (along c-axis) is justified by the difference in chemical binding along different crystallographic directions in a layer crystal.

We use two-time retarded Green's function $\left\langle\left\langle a_{0} \mid a_{0}^{+}\right\rangle\right\rangle$for intercalate. The equation of motion for operator $a_{0}$ is of the form

$$
\mathrm{i} \frac{\partial a_{0}}{\partial t}=\left[a_{0}, H\right]=V_{0} \sum_{\vec{\chi} k} c_{\vec{\chi} k}\left\{\mathrm{e}^{\mathrm{i}\left(\vec{\chi} \vec{n}_{0}+k l\right)}+\mathrm{e}^{\mathrm{i}\left(\vec{\chi} \vec{n}_{0}+k(l+1)\right)}\right\}+\varepsilon_{0} a_{0} .
$$

Then

$$
\begin{aligned}
\omega\left\langle\left\langle a_{0} \mid a_{0}^{+}\right\rangle\right\rangle= & 1+\varepsilon_{0}\left\langle\left\langle a_{0} \mid a_{0}^{+}\right\rangle\right\rangle \\
& +V_{0} \sum_{\vec{\chi} k}\left\langle\left\langle c_{\vec{\chi} k} \mid a_{0}^{+}\right\rangle\right\rangle\left\{\mathrm{e}^{\mathrm{i}\left(\vec{\chi} \vec{n}_{0}+k l\right)}+\mathrm{e}^{\mathrm{i}\left(\vec{\chi} \vec{n}_{0}+k(l+1)\right)}\right\}, \\
\left\langle\left\langle a_{0} \mid a_{0}^{+}\right\rangle\right\rangle= & \frac{V_{0}}{\omega-\varepsilon_{\vec{\chi} k}}+\frac{V_{0}}{\omega-\varepsilon_{0}} \sum_{\vec{\chi} k}\left\langle\left\langle c_{\vec{\chi} k} \mid a_{0}^{+}\right\rangle\right\rangle \mathrm{e}^{\mathrm{i} \vec{\chi} \vec{n}_{0}} \mathrm{e}^{\mathrm{i} k l}\left(1+\mathrm{e}^{\mathrm{i} k}\right) .
\end{aligned}
$$

From the equation of motion for the Green function $\left\langle\left\langle c_{\vec{\chi} k} \mid a_{0}^{+}\right\rangle\right\rangle$, we obtain

$$
\begin{aligned}
\left\langle\left\langle c_{\vec{\chi} k} \mid a_{0}^{+}\right\rangle\right\rangle= & \frac{V_{0}}{\omega-\varepsilon_{\vec{\chi} k}} \mathrm{e}^{-\mathrm{i} \vec{\chi} \vec{n}_{0}} \mathrm{e}^{-\mathrm{i} k l}\left(1+\mathrm{e}^{-\mathrm{i} k}\right)\left\langle\left\langle a_{0} \mid a_{0}^{+}\right\rangle\right\rangle \\
& -\frac{t-\gamma}{\omega-\varepsilon_{\vec{\chi} k}}\left[\mathrm{e}^{-\mathrm{i} k l} \Phi_{1}+\mathrm{e}^{-\mathrm{i} k(l+1)} \Phi_{2}\right],
\end{aligned}
$$

where

$$
\begin{aligned}
\Phi_{1} & =\sum_{k}\left\langle\left\langle c_{\vec{\chi} k} \mid a_{0}^{+}\right\rangle\right\rangle \mathrm{e}^{\mathrm{i} k(l+1)}, \\
\Phi_{2} & =\sum_{k}\left\langle\left\langle c_{\vec{\chi} k} \mid a_{0}^{+}\right\rangle\right\rangle \mathrm{e}^{\mathrm{i} k l} .
\end{aligned}
$$


Expression (5) is an integral equation with degenerated kernel. Thus, we have

$$
\left\langle\left\langle c_{\vec{\chi} k} \mid a_{0}^{+}\right\rangle\right\rangle=\frac{V_{0} \mathrm{e}^{-\mathrm{i} \vec{\chi}_{0}} \mathrm{e}^{-\mathrm{i} k l}\left(1+\mathrm{e}^{\mathrm{i} k}\right)\left\langle\left\langle a_{0} \mid a_{0}^{+}\right\rangle\right\rangle}{\left(\omega-\varepsilon_{\vec{\chi} k}\right)\left[1+(t-\gamma) S_{0}\right]},
$$

where

$$
S_{0}=\sum_{k} \frac{1+\cos k}{\omega-\varepsilon_{\vec{\chi} k}} .
$$

Using (6) and (4) we obtain

$$
\left\langle\left\langle a_{0} \mid a_{0}^{+}\right\rangle\right\rangle=\left[\omega-\varepsilon_{0}-2 V_{0}^{2} \sum_{\vec{\chi}} \frac{S_{0}}{1+(t-\gamma) S_{0}}\right]^{-1} .
$$

From the equation of motion for $\left\langle\left\langle c_{\vec{\chi} k} \mid c_{\vec{s}}^{+}\right\rangle\right\rangle$, where $\vec{s}$ is three-dimensional vector,

$$
\begin{aligned}
\left\langle\left\langle c_{\vec{\chi} k} \mid c_{s}^{+}\right\rangle\right\rangle= & \frac{\delta_{\vec{\chi} k, s}}{\omega-\varepsilon_{\vec{\chi} k}}+\frac{V_{0}}{\omega-\varepsilon_{\vec{\chi} k}} \mathrm{e}^{-\mathrm{i} \vec{\chi} \vec{n}_{0}} \mathrm{e}^{-\mathrm{i} k l}\left(1+\mathrm{e}^{-\mathrm{i} k}\right)\left\langle\left\langle a_{0} \mid c_{s}^{+}\right\rangle\right\rangle \\
& -\frac{(t-\gamma)}{\omega-\varepsilon_{\vec{\chi} k}}\left[F_{1} \mathrm{e}^{-\mathrm{i} k l}+F_{2} \mathrm{e}^{-\mathrm{i} k(l+1)}\right]
\end{aligned}
$$

with

$$
\begin{aligned}
& F_{1}=\sum_{k}\left\langle\left\langle c_{\vec{\chi} k} \mid c_{s}^{+}\right\rangle\right\rangle \mathrm{e}^{\mathrm{i} k(l+1)}, \\
& F_{2}=\sum_{k}\left\langle\left\langle c_{\vec{\chi} k} \mid c_{s}^{+}\right\rangle\right\rangle \mathrm{e}^{\mathrm{i} k l} .
\end{aligned}
$$

Equation of motion for $\left\langle\left\langle a_{0} \mid c_{s}^{+}\right\rangle\right\rangle$gives

$$
\left\langle\left\langle a_{0} \mid c_{\vec{s}}^{+}\right\rangle\right\rangle=\frac{V_{0}}{\omega-\varepsilon_{0}} \sum_{\vec{\chi}} \mathrm{e}^{\mathrm{i} \vec{\chi} \vec{n}_{0}}\left(F_{1}+F_{2}\right)
$$

and thus

$$
\begin{aligned}
&\left\langle\left\langle c_{\vec{\chi} k} \mid c_{\vec{s}}^{+}\right\rangle\right\rangle= \frac{1}{\omega-\varepsilon_{\vec{\chi} k}}+\frac{2 V_{0}^{2}(1+\cos k)}{\left(\omega-\varepsilon_{\vec{\chi} k}\right)^{2}\left(\omega-\varepsilon_{0}\right)\left[1+(t-\gamma) S_{0}\right]\left[1-2 \sum_{\vec{\chi}} A_{12}\right]} \\
&-\frac{2(t-\gamma)}{\left(\omega-\varepsilon_{\vec{\chi} k}\right)^{2}}\left[\frac{\cos k}{S}+\frac{V_{0}^{2}(t-\gamma)(1+\cos k) S_{0}}{\left(\omega-\varepsilon_{0}\right)\left[1+(t-\gamma) S_{0}\right]^{2}\left[1-2 \sum_{\vec{\chi}} A_{12}\right]}\right. \\
&\left.+\frac{(t-\gamma)\left(S_{1} \cos k-S_{2}\right)}{S}\right]
\end{aligned}
$$


with

$$
\begin{aligned}
S & =\left[1+(t-\gamma) S_{0}\right]\left[1+(t-\gamma)\left(S_{1}-S_{2}\right)\right] ; \\
S_{1} & =\sum_{k} \frac{\mathrm{e}^{\mathrm{i} k}}{\omega-\varepsilon_{\vec{\chi} k}} ; \quad S_{2}=\sum_{k} \frac{1}{\omega-\varepsilon_{\vec{\chi} k}} ; \\
A_{12} & =V_{0}^{2} \frac{S_{0}}{\left(\omega-\varepsilon_{0}\right)\left[1+(t-\gamma) S_{0}\right]} ; \\
S_{0} & =\sum_{k} \frac{1+\cos k}{\omega-\varepsilon_{\vec{\chi} k}+\mathrm{i} \delta}=P \sum_{k} \frac{1+\cos k}{\omega-\varepsilon_{\vec{\chi} k}}-\mathrm{i} \pi \sum_{k} \delta\left(\omega-\varepsilon_{\vec{\chi} k}\right)(1+\cos k) \\
& =\operatorname{Re} S_{2}+\operatorname{Re} S_{1}+\mathrm{i}\left(\operatorname{Im} S_{2}+\operatorname{Im} S_{1}\right) .
\end{aligned}
$$

In the case $t=\gamma$

$$
\begin{aligned}
\left\langle\left\langle c_{\chi k} \mid c_{\chi k}^{+}\right\rangle\right\rangle & =\frac{1}{\omega-\varepsilon_{\chi k}}+\frac{2 V_{0}^{2}(1+\cos k)}{\left(\omega-\varepsilon_{\chi k}\right)^{2}\left(\omega-\varepsilon_{0}\right)\left[1-2 \sum_{\vec{\chi}} A_{12}\right]}, \\
\left\langle\left\langle a_{0} \mid a_{0}^{+}\right\rangle\right\rangle & =\left[\omega-\varepsilon_{0}-2 V_{0}^{2} \sum_{\vec{\chi}} S_{0}\right]^{-1}, \\
A_{12} & =V_{0}^{2} \frac{S_{0}}{\left(\omega-\varepsilon_{0}\right)},
\end{aligned}
$$

For $V_{0}=0$

$$
\left\langle\left\langle c_{\vec{\chi}} k \mid c_{\vec{\chi} k}^{+}\right\rangle\right\rangle=\frac{1}{\omega-\varepsilon_{\vec{\chi} k}}-\frac{2(t-\gamma)}{\left(\omega-\varepsilon_{\vec{\chi} k}\right)^{2}}\left[\frac{\cos k\left[1+(t-\gamma) S_{1}\right]}{S}-\frac{(t-\gamma) S_{2}}{S}\right] .
$$

\section{Calculations}

Density of electron states of intercalated LC is found from imaginary part of Green's function

$$
\rho_{m}(\omega)=-\frac{1}{\pi} \operatorname{Im}_{\varepsilon \rightarrow 0}\left\langle\left\langle a_{m} \mid a_{m}^{+}\right\rangle\right\rangle_{\omega+\mathrm{i} \varepsilon} .
$$

To avoid bulky expressions, the density of electron states of intercalated LC and intercalate is given here only for the case $t=\gamma$

$$
\begin{aligned}
\rho_{\mathrm{LC}}(\omega)=\rho_{0}(\omega) & +\frac{2 V_{0}^{2}}{\pi\left(\omega-\varepsilon_{0}\right)} \sum_{\vec{\chi} k} \frac{1+\cos k}{\left(\omega-\varepsilon_{\vec{\chi} k}\right)^{2}} \\
\times & \frac{2 V_{0}^{2} \sum_{\vec{\chi}} \operatorname{Im} S_{0}}{\left(\omega-\varepsilon_{0}\right)\left[1-2 \frac{V_{0}^{2}}{\left(\omega-\varepsilon_{0}\right)} \sum_{\vec{\chi}} \operatorname{Re} S_{0}\right]^{2}+\left[2 \frac{V_{0}^{2}}{\left(\omega-\varepsilon_{0}\right)} \sum_{\vec{\chi}} \operatorname{Im} S_{0}\right]^{2}},
\end{aligned}
$$


where

$$
\rho_{0}(\omega)= \begin{cases}\frac{2 \pi}{\alpha} \arccos \frac{2 t-\omega}{2 t}, & \omega \leqslant 2 t \\ \frac{\pi^{2}}{\alpha}, & \omega \geqslant 2 t\end{cases}
$$

and

$$
\rho_{i}=\frac{1}{\pi} \frac{2 V_{0}^{2} \sum_{\chi} \operatorname{Im} S_{0}}{\left[\omega-\varepsilon_{0}-2 V_{0}^{2} \sum_{\chi} \operatorname{Re} S_{0}\right]^{2}+\left[2 V_{0}^{2} \sum_{\chi} \operatorname{Im} S_{0}\right]^{2}} .
$$

Density of states may be presented by $\rho_{\mathrm{LC}}=\rho_{0}+\Delta \rho$, where $\rho_{0}=\rho_{p}+\rho^{(i)}\left(\rho_{p}\right.$ and $\rho^{(i)}$ are densities of states of pure LC and intercalant, respectively), $\Delta \rho$ describes the distortion in density caused by interaction between LC and intercalant. We neglect the contribution of the renormalized intercalate density of states which in the one-impurity case is of $O(1 / N)$ order, $N$ is the number of crystal sites.

The change of free energy $\Delta F$, caused by intercalation at $T=0$ equals to

$$
\Delta F=\int_{0}^{\varepsilon_{\mathrm{F}}} \rho_{\mathrm{LC}}(\omega) \omega \mathrm{d} \omega-\int_{0}^{\varepsilon_{\mathrm{F}}} \rho_{0}(\omega) \omega \mathrm{d} \omega=\int_{0}^{\varepsilon_{\mathrm{F}}} \Delta \rho(\omega) \omega \mathrm{d} \omega,
$$

where $\omega$ is counted off from the conduction band bottom. We calculated $\Delta F$ numerically depending on: (a) $\varepsilon_{\mathrm{F}}$, (b) degree of the electron intercalate-LC mixing, $V_{0}$ (which was changed in the range $0.05-0.15 \mathrm{eV}$; the magnitude of energy parameter $V_{0}$ was chosen according to the results of the calculations of resonance levels and occupancies of an atom or molecule on the crystal surface carried out in terms of a model Hamiltonian of Anderson's type [9]), (c) changes of the electron hopping constant along c-axis due to intercalation, $t-\gamma$. As it was shown in [10], intercalation can be accompanied by either the increase or the decrease of the latter parameter. Therefore $t-\gamma$ is negative in the first case and positive in the second one $(|t-\gamma| \leqslant 0.04 \mathrm{eV})$, while the different impurities were considered as intercalant. Hence, their energetic position was changed in the range $-0.2 \leqslant \varepsilon_{0} \leqslant 0.2(\mathrm{eV})$ (value $t=0.1 \mathrm{eV}$ was chosen), including special points in density of electron states of ideal LC; point A was the fold point and point B was the point of non-analyticity (figure 1).

\section{Discussion}

As it follows from figure 2, at fixed $V_{0}$ and $t-\gamma, \Delta F$ is a nonmonotonic function and the higher is $\varepsilon_{\mathrm{F}}$ the higher $\Delta F$ will be. At $\varepsilon_{0}=10^{-5} \mathrm{eV}, \Delta \mathrm{F}$ is negative only at $\varepsilon_{\mathrm{F}} \leqslant 0.002 \mathrm{eV}$. Dependence of $\Delta F=f\left(\varepsilon_{\mathrm{F}}\right)<0$ for $\varepsilon_{0}>10^{-5} \mathrm{eV}$ has the similar type independently on $\varepsilon_{\mathrm{F}}$, the peak is shifted in the direction of higher $\varepsilon_{\mathrm{F}}$ at $t-\gamma$ increase. If the intercalate does not change electron mixing between atoms from the nearest layers $(t-\gamma=0) \Delta F$ is almost monotonous weakly increasing $\varepsilon_{\mathrm{F}}$ function with a small peak at $\varepsilon_{\mathrm{F}}=0.03 \mathrm{eV}$ (figure 2). 


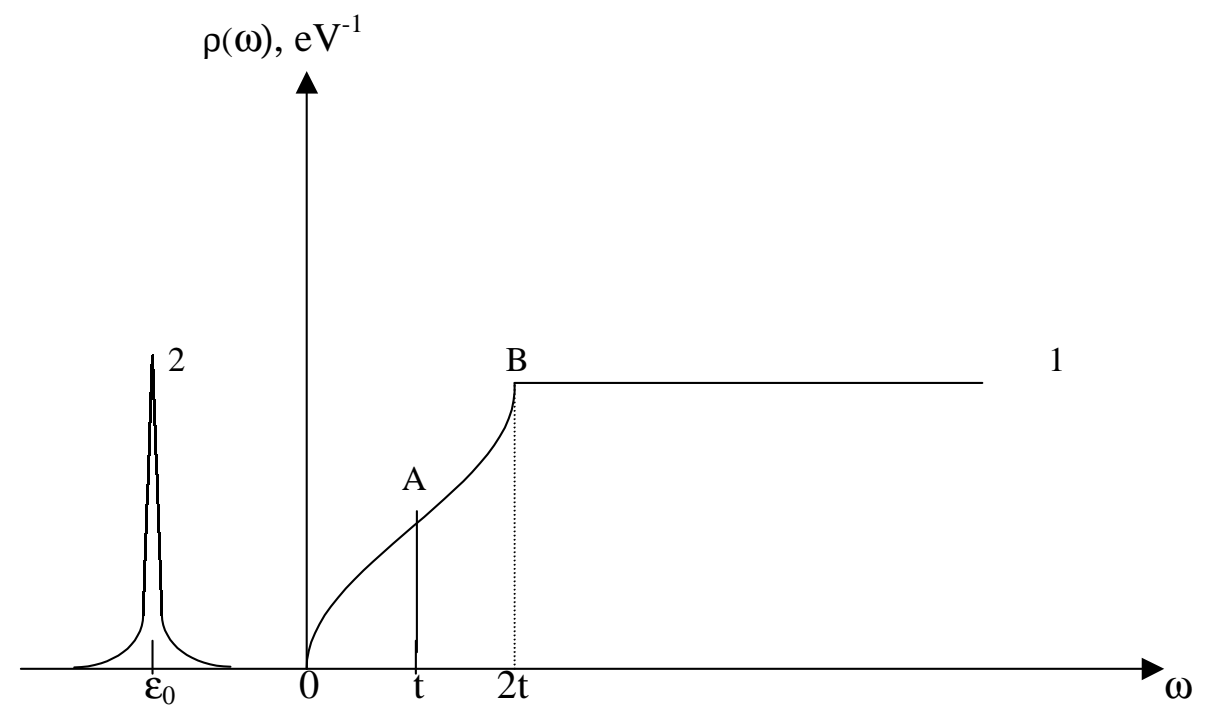

Figure 1. Schematic density of states of the intercalant-crystal system: 1 is density of states of ideal LC $\rho_{0}, 2 t$ is bandwidth along anisotropy axis; 2 is the position of intercalate energy level $\varepsilon_{0}$.

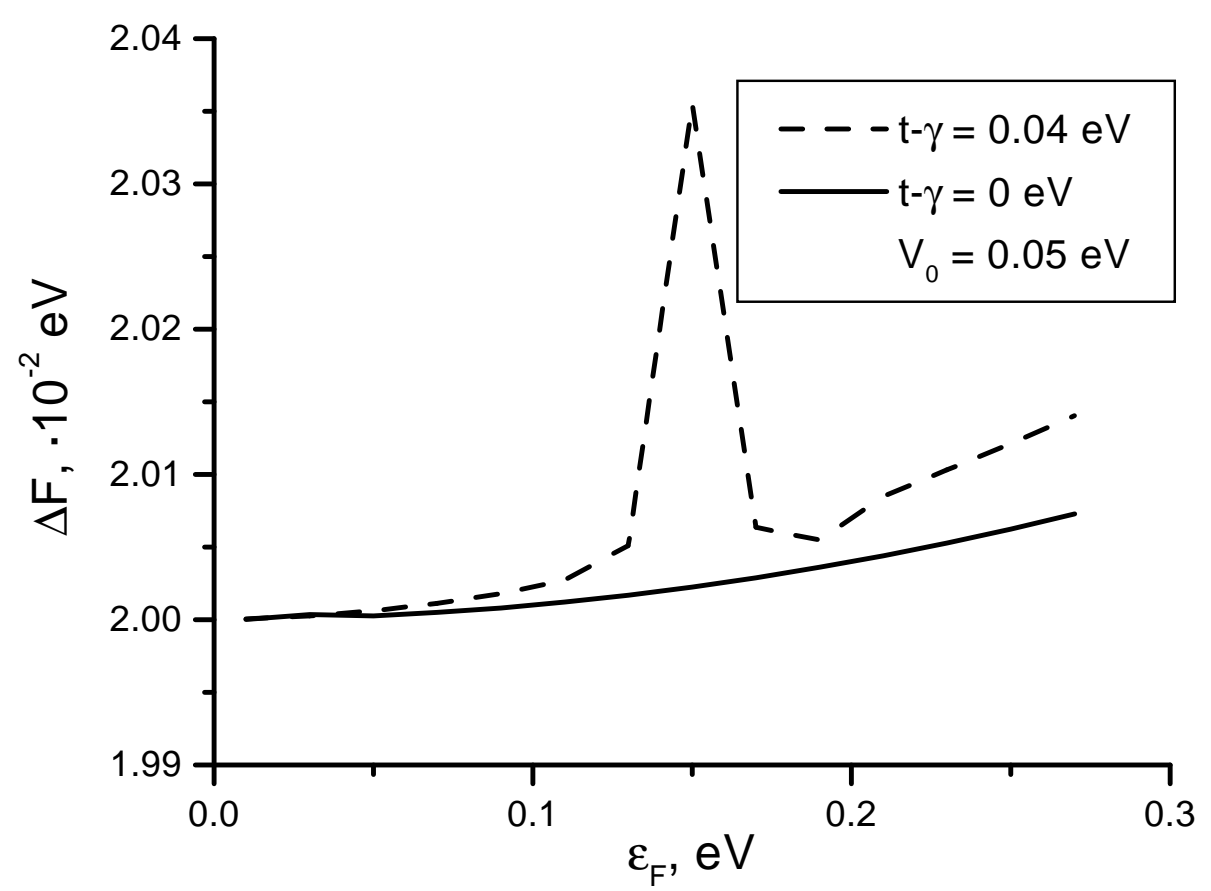

Figure 2. Thermodynamic potential changes for the systems with different $\varepsilon_{\mathrm{F}}$ at $\varepsilon_{0}=-0.02 \mathrm{eV}$. 


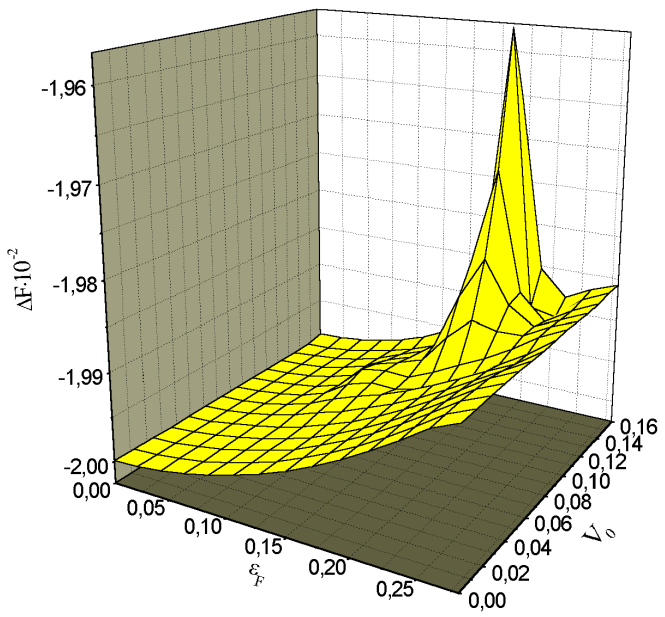

(a)

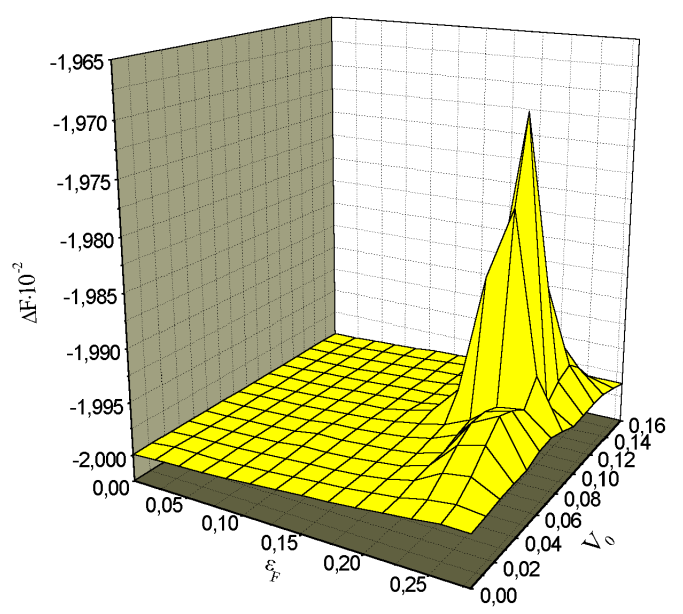

(b)

Figure 3. Thermodynamic potential change (in $\mathrm{eV}$ ) for the systems with different $\varepsilon_{\mathrm{F}}$ depending on $V_{0}$ : (a) $t-\gamma=0.04 \mathrm{eV}$, (b) $t-\gamma=-0.04 \mathrm{eV} ; \varepsilon_{0}=0.02 \mathrm{eV}$.

Comparison of $\Delta F=f\left(\varepsilon_{\mathrm{F}}\right)$ dependences for different $V_{0}$ and $\varepsilon_{\mathrm{F}} \in[0 \div 0.23] \mathrm{eV}$ (figures $3 \mathrm{a}, 3 \mathrm{~b}$ ) shows the existence of two intervals of $V_{0}$, at which instead of the monotonous increase, a peak is observed. In the case of anisotropy increase $(t-\gamma=$ $0.04 \mathrm{eV}$ ) the monotonic dependence takes place when $V_{0} \in[0 \div 0.1] \mathrm{eV}$ (figure 3a), whereas for $t-\gamma=-0.04 \mathrm{eV}$ such a dependence is observed for $V_{0} \in[0 \div 0.03] \mathrm{eV}$ (figure $3 \mathrm{~b}$ ).

At $t-\gamma=-0.04 \mathrm{eV}$ and $V_{0}=0.05 \mathrm{eV}, \Delta F=f\left(\varepsilon_{\mathrm{F}}\right)$ has two maxima. At $t-\gamma$ increase, the second maximum vanishes, and the first one increases the shifting to the large values of $\varepsilon_{\mathrm{F}}$ decrease (figure 4 ). For positive $t-\gamma$, only one peak near $\varepsilon_{\mathrm{F}}=0.05 \mathrm{eV}$ exists, it vanishes at $t-\gamma=0.04 \mathrm{eV}$ and $\Delta F=f\left(\varepsilon_{\mathrm{F}}\right)$ becomes a monotonic function.

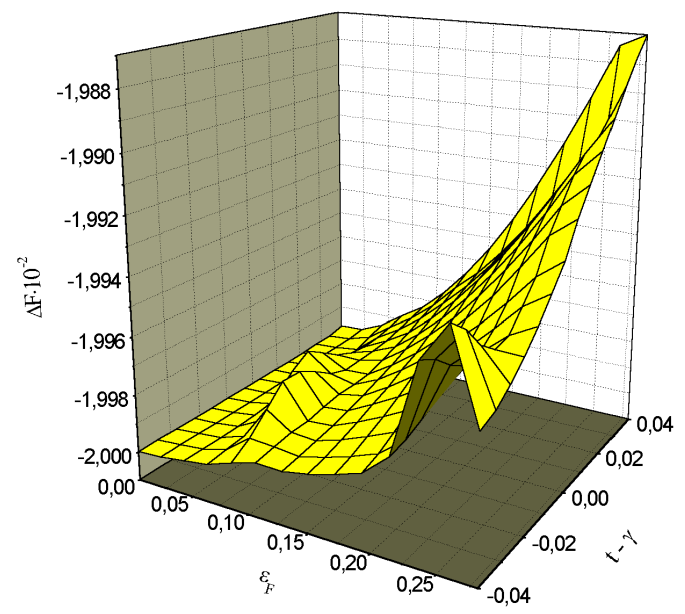

Figure 4. Thermodynamic potential change (in $\mathrm{eV}$ ) for the systems with different $\varepsilon_{\mathrm{F}}$ depending on $t-\gamma$ at $V_{0}=0.05 \mathrm{eV}, \varepsilon_{0}=0.02 \mathrm{eV}$. 
The existence of maxima $\Delta F$ at $\varepsilon_{\mathrm{F}} \approx 0.1 \mathrm{eV}, 0.23 \mathrm{eV}$ (figure 4) permits to determine the range of parameter values for which the intercalation-deintercalation process is the most stable.

\section{Conclusions}

Calculations of $\Delta F$ as a function of $\varepsilon_{\mathrm{F}}$ at certain $V_{0}$ and $t-\gamma$ show two intervals of $V_{0}$ values for which $\Delta F$ increases with $\varepsilon_{\mathrm{F}}$ (a) in the monotonic way; (b) with peaks. In the case of anisotropy increase $(t-\gamma>0)$, a monotonic region occurs at $V_{0} \in[0 \div 0.1] \mathrm{eV}$, for the anisotropy decrease - at $V_{0} \in[0 \div 0.03] \mathrm{eV}$ correspondingly.

It is shown that thermodynamic stability (that is $\Delta F<0$ ) of the intercalated LC occurs in case:

- When intercalant electron level is in the conduction band, $|\Delta F|$ is growing, being however still negative if $\varepsilon_{\mathrm{F}}$ increases;

- The behaviour of $\Delta F$ with the change of $\varepsilon_{\mathrm{F}}$ is different, it depends on anisotropy increase or decrease.

\section{Acknowledgements}

This work was supported by Science Technology Center in Ukraine under project No. 818.

The author would like to thank professor B.A.Lukiyanets for the numerous stimulating discussions.

\section{References}

1. Safran S.A. Stage ordering in intercalation compounds. // Solid State Phys., 1987, vol. 40, p. 183-247.

2. Grygorchak I.I., Gavrylyuk S.V., Netyagha V.V., Kovalyuk Z.D. Structure and physical properties of InSe, GaSe, selectively intercalated by Li. // Journal of Physical Studies, 2000, vol. 4, No. 1, p. 82-84.

3. Ivanov-Omskij V.I., Smorgonskaja E.A. Charge shift at intercalation of carbon-like nanoclusters in amorphous carbon by Cu. // Fizika Tverdogo Tela, 1999, vol. 41, No. 5, p. 868-870 (in Russian).

4. Koshkin V., Dmitriev Yu. Chemistry and physics of compounds with loose crystal structure. // Chem. Reviews, 1994, vol. 19, p. 1-138.

5. Grygorchak I.I. Thermodynamic aspects of layer crystal intercalation. - In: Material Science of Narrow Gap and Layer Semiconductors. Kyjiv, Naukova Dumka, 1989, p. $65-70$.

6. Liang W.Y. Electronic properties of transition metal dichalcogenides and their intercalation complexes. - In: Proc. 10 Course Erive Summer School, 1986.

7. Lukiyanets B., Tovstyuk N. Electron density state of intercalated layer crystal. // Mol. Phys. Rep., 1999, vol. 23, p. 162-166. 
8. Lukiyanets B.A., Tovstyuk N.K. Density of electron states of intercalated layer crystal. // Ukr. J. Phys., 2001, vol. 46, No. 1, p. 100.

9. Grimley T.B. Electronic structure of adsorbed atoms and molecules. // Journal of Vacuum Science and Technology, 1972, vol. 8, No. 1, p. 31-38.

10. Mustafayeva S.N. // Izv. RAN Neorgan. Material., 1994, vol. 30, p. 1033 (in Russian).

\section{Термодинаміка інтеркальованих шаруватих кристалів при низьких температурах}

\section{Н.К.Товстюк}

Львівський національний університет ім. І.Франка, фізичний факультет, 79005 Львів, вул. Драгоманова, 50

Отримано 23 квітня 2001 р.

На основі моделі типу моделі Андерсона отримано густину електронних станів інтеркальованого шаруватого кристалу. Досліджується термодинамічна стабільність кристалу залежно від ширини дозволеної зони, ступеня шаруватості, енергетичного стану інтеркалянта i викликаної інтеркаляцією зміни параметра електронного перемішування.

Ключові слова: термодинаміка, інтеркальовані шаруваті кристали, інтеркалянт, інтеркалант

PACS: $65.90 .+i, 71.20 . T$ 